\title{
GAME AS TEXT AS GAME: THE COMMUNICATIVE EXPERIENCE OF DIGITAL GAMES
}

\author{
Carlos Magno Camargos Mendonça \& Filipe Alves de Freitas
}

\begin{abstract}
We propose to regard video game as text, but not by literally understanding it as a verbal expression, and instead recognizing that many assumptions of literary theory are relevant to its analysis. This option seems to put us in sync with the narratologists, who exalt games as new manifestations of narrative, but cling to a conception of text as world that values illusionist effects. Instead, we are interested in experiences that, against this perspective, recognize the possibility of regarding game as a text that is a game - an incomplete object that is to be updated by the reader in a self-reflective relationship with the signs that compose it, a central notion to theories such as Iser's and Dewey's. Then, instead of focusing on strategies of immersion on large virtual worlds, we favor small independent casual games (such as Small Worlds, Grey, The Beggar, and Dys4ia) analyzing how, in these, take place experiences that allow us to re-examine the aesthetic potential of the medium.
\end{abstract}

\section{KEYWORDS}

Text; aesthetic experience; immersion; performance

\section{INTRODUCTION}

"There is too much noise", a caption tells us. The pixelated scene that follows seems to confirm the statement: it lacks clarity and requires effort to be deciphered. We need a few seconds to realize that three colored squares suggest the form of a person standing in the center of a transparent dome, in a gloomy setting.

This is the opening scene of Small Worlds, game submitted by David Shute for the sixth edition of the Casual Gameplay Design Competition, whose theme was "exploration". The work's concept in principle seems straightforward: using the arrow keys, the player controls a character in a setting that becomes visible as it is traversed. One of the distinctive aspects of the game is the way it looks: it seems to evoke the graphic style of the first low-resolution games, built with a limited number of pixels. Little information is given about the story and background: after the initial caption the player is set to explore a setting that may be a ruined lab or space station. As he explores new areas, the field of vision expands, as if a virtual camera retreated. Thus, the "pixels" (and with them the character) decrease in size on the screen, resulting in a higher resolution - the relationship between the number of pixels and the screen area - and the scenery gradually becoming clearer (Figure 1). At the bottom of the stage four colored "portals" can be found, objects that, when touched by the avatar, transport it to other "worlds" (Figure 2), where the same logic of gradual exploration reigns. 
Small Worlds won the jury prize and was chosen by the audience of the site as the best game of the competition, being also voted the best game of 2009 by collaborators of jayisgames.com. On the website page dedicated to it, users have fun exchanging interpretations about the game's narrative, but it is also interesting to discuss its strategies of use and dialogue with the matter of the medium. It is undeniable that the pixelated graphics are one of the essential elements of this material, as they invoke a style typical of games that was appropriated by art, in the form of so-called "Pixel Art". With the spread of digital technology, the pixel — short for picture element - became a basic component of the contemporary experience of media and was converted into an essential unit of scale, something that becomes particularly evident in Small Worlds. The "small worlds" from Shute's game could easily occupy a fraction the size of a fingernail of the area of latest devices' high definition screens.

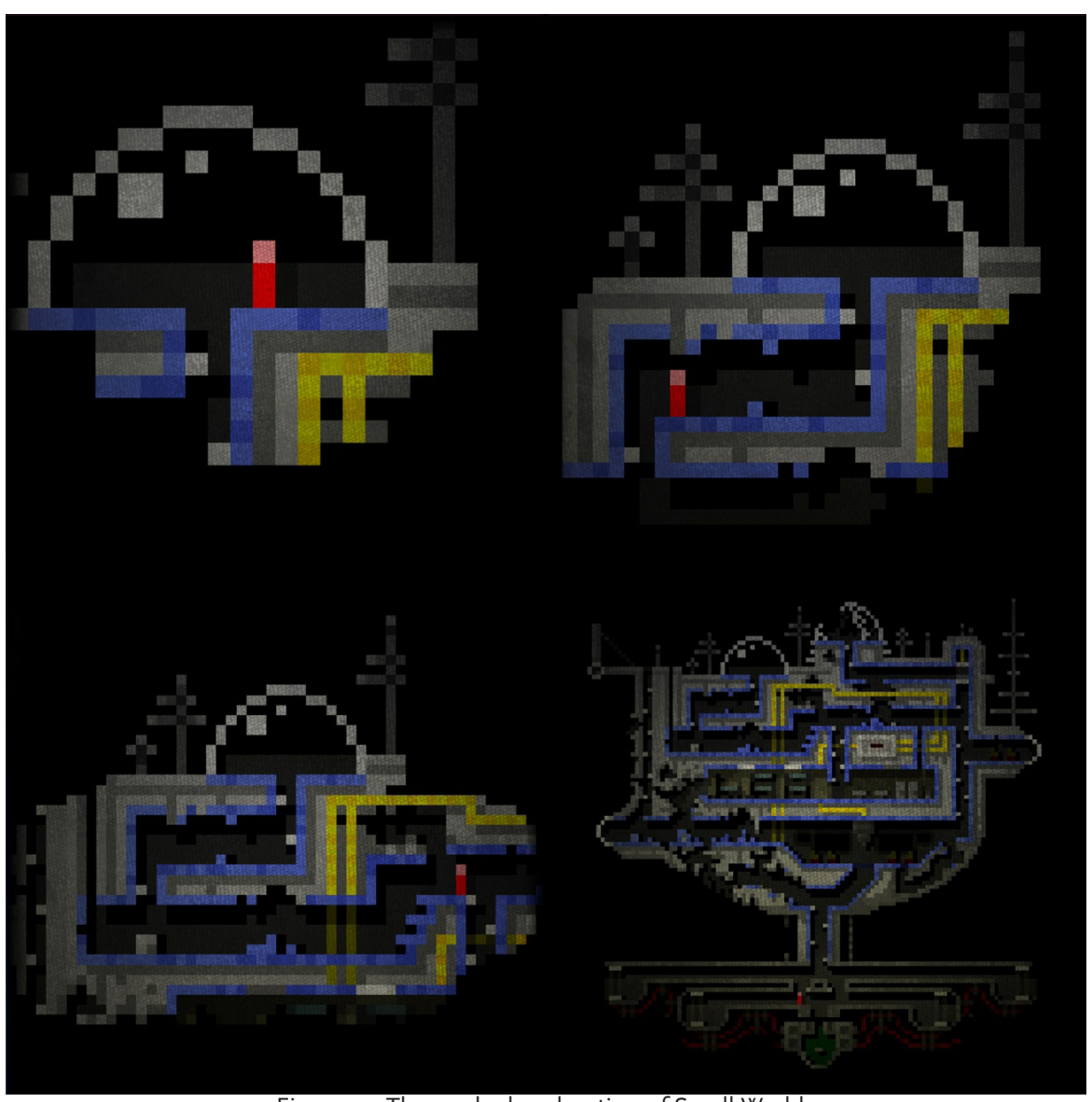

Figure 1 - The gradual exploration of Small Worlds

Maybe that's what the author suggests when he starts the game with the phrase "there's too much noise." In the "HD Era", the pixel became devalued, since it can only be used in large quantities. In the video game industry, its devaluation began even earlier: already in the 1980 s came the first vector graphics games. In traditional digital images, also known as raster graphics, the screen was drawn by scanning in horizontal rows of pixels; in vector graphics, the image is drawn on the screen as traces connecting points. With this technology game graphics have gained more clarity, but game art was no longer created pixel by pixel. This tendency was strengthened with the emergence of computer 
graphics and three-dimensional images constructed with vertices and polygons, reducing the pixel to mere hardware limitation, the resolution of the video display.

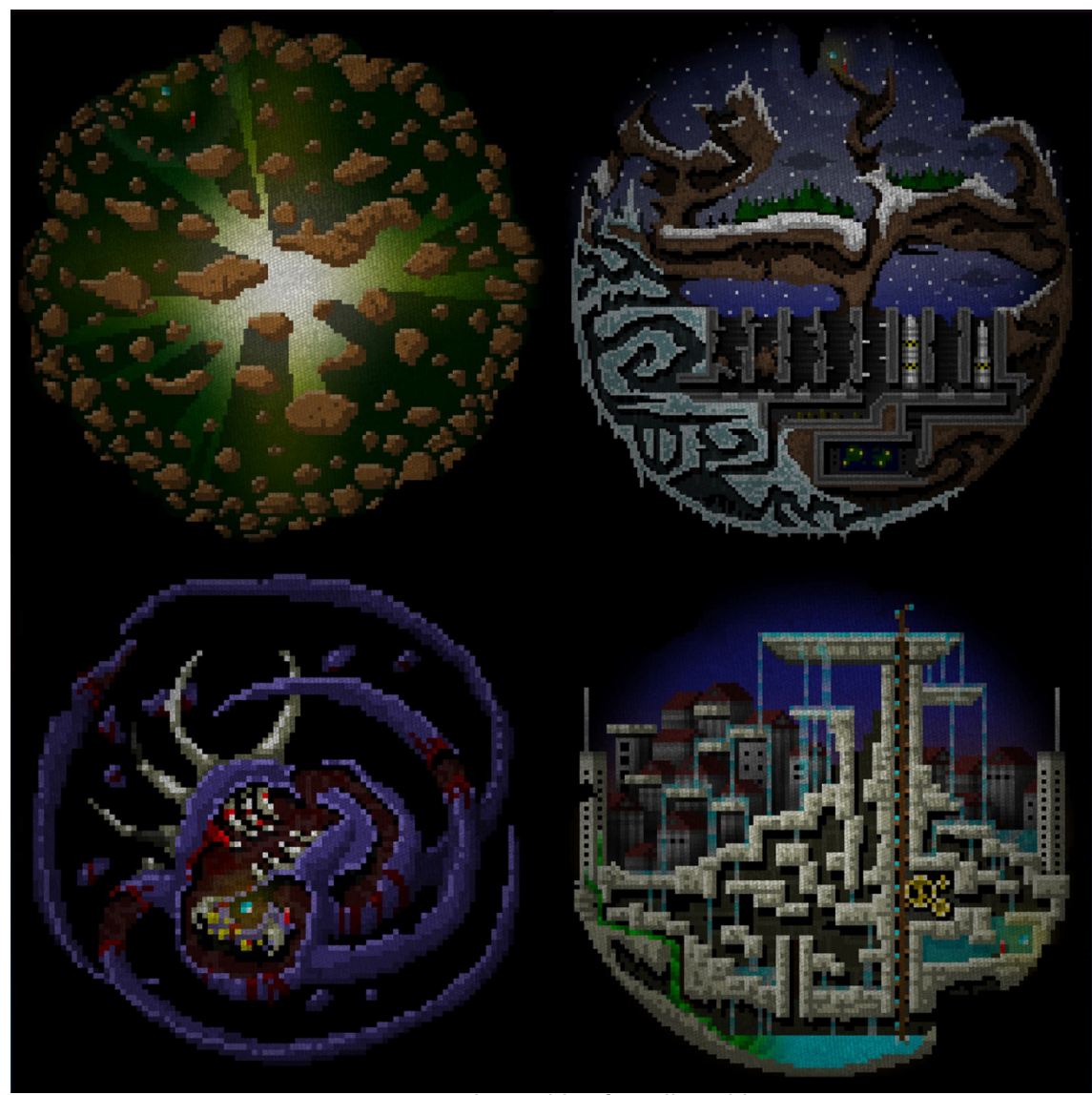

Figure 2 - The worlds of Small Worlds

Small Worlds would then be an ode to the raster graphics of the first decades of video game history, a "simpler" time in contrast to the overly complex images of the latest games. However, it is not about mere nostalgia. The rescue of low-resolution graphics in Small Worlds isn't just a manifestation of the longing for the games that many adults of today have experienced in their childhoods, but a resumption of an important element of graphic art and video games: the technical limitation.

The hypothesis that the artist finds his ingenuity by confronting the limitations imposed by the material of the medium in which he creates is proposed by Luigi Pareyson (1997). He suggests that artistic creation operates as a "dialogue with the matter", and deducts from this that art doesn't consist in the successful application of learned techniques or skills, but in a continuous historical process of inventing ways of use for the matter. Therefore, we could only conceive art as "language" metaphorically: while there are arts that embrace language as a subject, like poetry, there are also others, such as sculpture and architecture, which should be understood not as a result of the application of an organized system such as language, but as the fruit of a productive activity, "a doing that, while doing, invents the to do and the how to do" (Pareyson, 1997: 26). So

\footnotetext{
' Free translation from the authors.
} 
Pareyson conceives the artist's activity as one in which a "language" is not given a priori, but developed during production from possibilities and resistances offered by the matter and only perceived by the artist in his confrontation with it.

At least with respect to the graphical aspect, today's video game faces few obstacles. By working with low resolutions and images built pixel by pixel, Shute brings the Pixel Art, the art of constructing images using blocks of pure color, back to the video game. It's the return of an obstacle that was overcome by technology, so it can once again be overcome by the artist: how to create compelling images with a limited number of square elements.

The mechanics of Small Worlds serve to revalue the techniques of Pixel Art. It's a different experience of the pixel than what we have today in HD devices: instead of devoting little attention to the individual pixel, quickly passing by a stream of high definition images, we are forced to ergodically explore the pictures, discovering the scenery pixel by pixel, gradually experiencing increasing sharpness, with a keen eye for the skill required to build this sharpness with so few elements.

Small Worlds is but one among many "small games", independent and experimental works that defy the conceptions sedimented in the criticism of the medium. In order for us to begin to understand the effects that these games produce in the player, without being confined only to the strong sense of nostalgia some of them evoke, we should seek to understand video game as a kind of text. Unlike many authors in the field who sought to do the same - most of which were interested in the possible uses of games as teaching aids - , we do not propose that games should be understood literally as verbal manifestations. This decision sets us apart from most studies of hypertexts or cybertexts - term coined by Espen Aarseth (1997), who would certainly oppose the wider and more metaphorical use of the word "text" suggested here. These studies generally understand "text" as a material vehicle that conveys information or meaning verbally, and treat games as special types of texts, emphasizing the particularities that distinguish them from other textual forms.

Instead we use "text" in a loose sense, less as a formal concept than as a starting point to advocate that many assumptions of literary theory can be applied to its analysis. In the field of studies on the topic, narratologists, who exalt digital games as new manifestations of narrative phenomena, do something similar, and our option seems to put us in sync with them. That's not quite the case, as we will argue.

\section{BREAKING IMMERSION}

In general, narratologists seem to operate, in their analysis, based upon the metaphor of the "world as text". They regard video games as virtual realities, taking the term "virtual" in the sense of "false" or "double", in which it bears an affinity with the concept of fiction, and are interested in the strategies through which the text/game produces this world in the reader/player's imagination. Thus they tend to favor effects such as immersion, usually understood as the intense experience of presence before the virtual world 
produced by the possibilities of interaction with the fictional environment and by a visually compelling representation. Immersion, then, presupposes a kind of illusionistic realism.

This appreciation of realistic effects in virtual environments is clear in the views of Janet Murray (2003), the most famous representative of this trend. Murray is the proponent of the notion of "cyberdrama", a concept of emergent interactive narrative that is only fully realizable through the fictional technology of the holodeck - an immersive environment populated by solid intelligent holograms, and that only exists in the futuristic universe of the Star Trek TV series.

Such fetishism of illusionist technology is detrimental to the study of the aesthetic effects of video games, since it may induce us to think, as Castañares (2007), that virtual reality aims at resuming a goal that art had pursued until the twentieth century, and since abandoned: to create illusions. He says that "the novelty of these new representations is that they intend, and some of their manifestations can reach, experiences that are similar to the perception of reality and not the interpretation of representations" ${ }^{2}$ (Castañares, 2007: 42).

For the narratologist Marie-Laure Ryan (2001), the illusionist realism upon which immersion depends in a "world of the text" seems in fact incompatible with the exercise of critical faculties. "For a reader to be caught up in a story," she notes, "the textual world should be accessible through effortless concentration" (Ryan, 2001: 96), something that could be likened to a sort of "semiotic blindness", since the represented world is perceived in such intensity that one does not see the signs that make it up. The problem, she explains, is that attention to the rhetorical devices by which a world emerges from the text is an essential aspect of aesthetic appreciation and one of the favorite effects of postmodern literary theory. Still, since no text is fully illusionist, she argues that there can be immersion and, at the same time, appreciation for the stylistic virtuosity which produces the effect of presence.

It is, however, a curious argumentative strategy by Ryan. We cannot help noticing that the notion that the primary aesthetic effect of the game would come from a recognition of the technical skills involved in creating the work refers to the classical concept of art as understood by the greeks: techné, the practical knowledge to manufacture objects (a house, statue or ship), or perform a task (such as commanding armies or persuading an audience), and that gave rise to the medieval expression ars, used since the Renaissance to refer to the skill in the production of beauty (Tatarkiewicz 1997: 39;51). Although this conception of art remains present in the common usage of the term, it has been challenged since the modernist movements. Instead of silencing critics such as Castañares, her argument seems to confirm the thesis that we would have in games and virtual realities a sort of aesthetic atavism.

The persistence of authors like Ryan and a great many theoreticians and professionals of the gaming industry in dealing with the aesthetic potential of games from a classical view of art may have something to do with the refusal of contemporary critics and curators to accept video game as an art form. Because of this, interest us those

\footnotetext{
${ }^{2}$ Free translation from the authors.
} 
experiences that, going against this trend of the industry and academia, recognize the possibility of regarding the game as a text that is a game - a text that is an incomplete object to be updated by the reader in a self-reflective relationship with the signs that make it up, a notion that is central to theories such as Wolfgang Iser's and John Dewey's, and that allow us to visualize a communicative process in which there is no common code given a priori. From this theoretical angle, we can put aside the usual concerns about the strategies of immersion on large virtual worlds and turn our attention to smaller, independent casual games, considering how in four of those games - Small Worlds, Grey, The Beggar and Dys4ia - take place communicative experiences that allow us to re-examine the aesthetic potential of this new medium.

\section{INTERRUPTING THE FLUX OF EVERYDAY EXPERIENCE}

In Kevin McGrath's Grey the player takes control of what appears to be a boy actually, just a stylized silhouette formed by two black rectangles (head and trunk), two white rectangles (eyes), and two traces (the legs). Early in the game the boy sits atop a platform, accompanied by a girl - a silhouette similar to the protagonist, except for the long hair. The girl is sitting still on the deck, staring at nothing, while the boy tries to get her attention, without success. From there the player takes over, and can use the arrow keys to move the character across the stage.

Grey follows some of the conventions of the "platform" genre, broadly characterized by a two-dimensional setting in which the player must take an avatar jumping between platforms to reach a certain goal. In the classic Donkey Kong (Nintendo, 1981), the player controlled the Jumpman (later named Mario), who had to jump over barrels thrown by a gorilla to rescue Pauline, his girlfriend held hostage by the villain. Many of the games in the genre follow a kind of narrative that refers to medieval chivalric romances in which the hero must rescue the damsel, defeat a monster or bring back a treasure to win the girl's heart. At first, this seems to be the case with Grey, when colorful arrows appear pointing toward objects placed all around the stage, items that may be collected by the player and taken to the platform in which the girl sits (Figure 3). There are seven objects whose colors correspond to the arrows pointing to them: a red balloon, a green clover, a blue necklace, a purple lollipop, a pink bowtie, an orange teddy bear, and a yellow flower. We can recognize these as objects that a boy would give a girl, so it's easy to assume that Grey's protagonist is trying to win his beloved's affection.

Contrary to the expectations of narratologists, the game obviously does not pursuit a realistic graphic style. The scenery includes stylized trees, houses and buildings, grouped into sections that sometimes resemble urban landscapes, and other times country scenes, but organized in platforms, land sections that seem to float in the air above each other. This may seem strange to a player unfamiliar with the genre, but it is a convention of platform games. Another "break" in relation to a supposed illusionism lies in the fact that the game's setting is presented in grayscale - hence the name. The only color elements, at first, are the arrows and the objects to which they are tied. Once a 
player takes an object to the girl on the platform, the object's color appears in the scenery: plants become green when the clover is delivered, bricks turn red after the balloon is taken to the girl, and so on (Figure 4). The scenery gradually colors itself, and also gains movement and life with the emergence of birds, butterflies and fish.
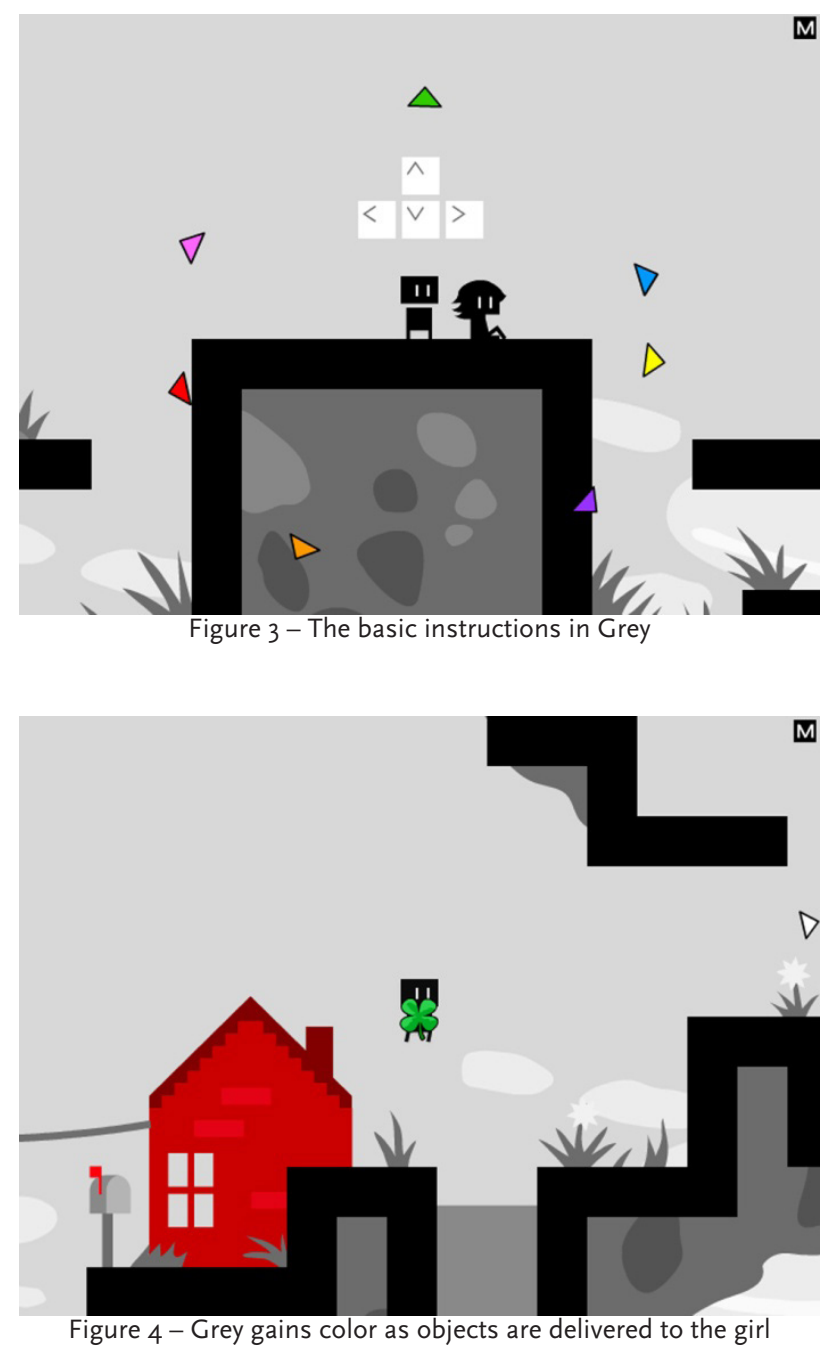

An analysis guided by narratological bias would see these breaks with an illusionist project as a sign of the developer's incompetence or adherence to an aesthetic "fad", which is to highlight the signs that make up the work to the detriment of an immersive effect. Authors such as Dewey (2010), however, allow us to see this kind of "break" as the trigger that starts the aesthetic experience, which he describes as "an" experience able to promote the rapture of the living creature from the continuous flow of everyday experience, thanks to a number of particular features: an establishing rupture, an organized development, an unifying element and a possibility of "consummation". In Dewey's conception, of Darwinian influence, these traits are interrelated: assuming that, in nature, the form is a result of stability, Dewey suggests that the organism always seeks to transform disturbance and conflict in stable relationships, which results in a consummation that has aesthetic quality (Dewey, 2010: 77). In other words, in the structure of Dewey's aesthetic experience we have a situation in which the organism is faced with a perceptual 
break, engages with the elements of experience interested in remedying this breach, calls elements of its own repertoire to reorganize these elements and discover possible new harmonious relations between them and thus resolves the conflict and reaches an aesthetic fulfillment.

In Grey, the rupture which the player faces is not just visual. The game also gradually "breaks" with the initial interpretation of its background narrative. Every time the player successfully delivers an object to the girl, his avatar becomes a bit more transparent and diffuse, becoming a little more than a shadow during the search for the last items (Figure 5). In addition, one of the items brings a disturbing indication of what will come next: a yellow flower is deposited, in one of the most remote sections of the setting, in front of a tombstone (Figure 6). When the last object is delivered to the girl, two things occur: the player's avatar disappears as if evaporating in the air and the girl catches in her arms a white bird, possibly a dove, and then releases it.

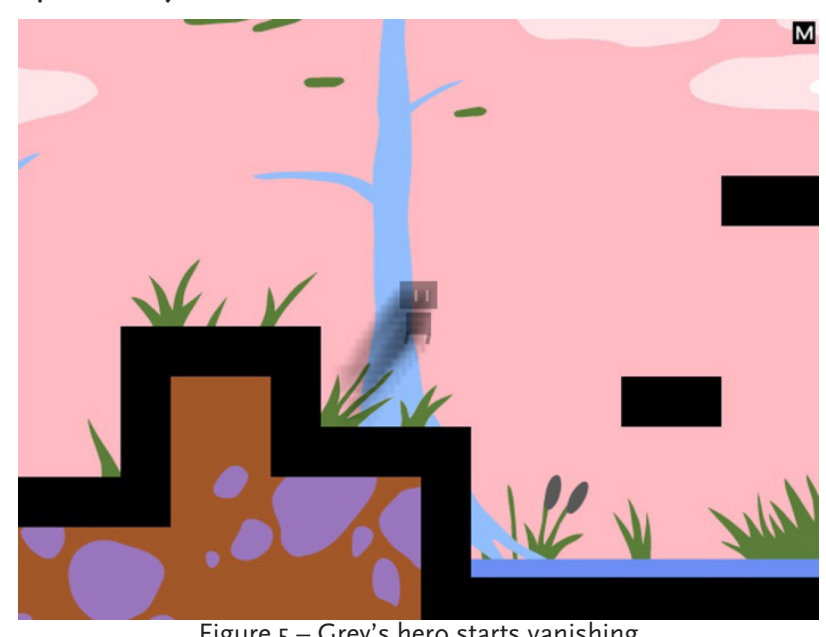

Figure 5-Grey's hero starts vanishing

Thus, after driving the player's expectation in one direction, Grey reverses it: the protagonist is no longer a young man trying to win his beloved, he's a ghost or a memo$r y$, and the girl, who may be his girlfriend, wife, mother or daughter, is someone who has lost their loved one. The game is no longer a chivalric romance converted into platform game, but a narrative about loss and overcoming grief.

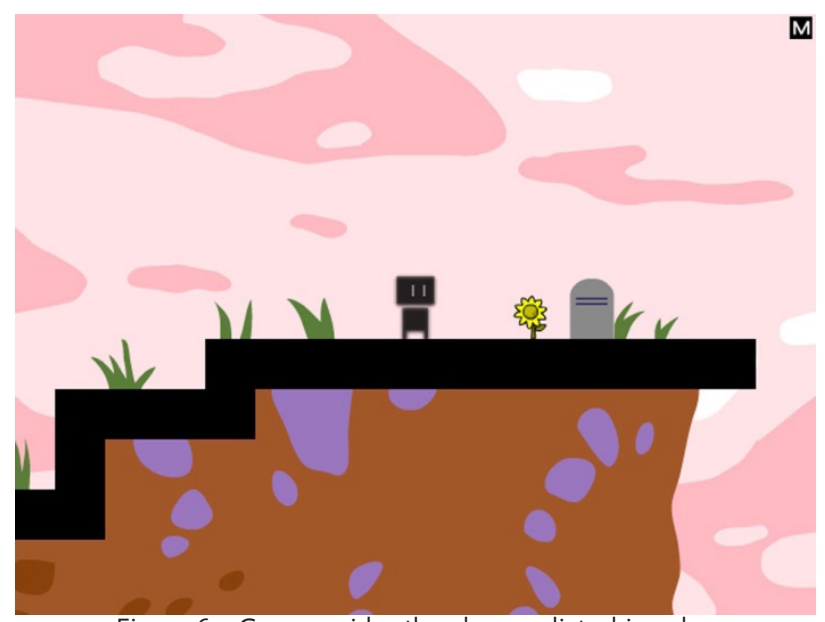

Figure 6 - Grey provides the player a disturbing clue 
This does not imply, however, a conception of the work as something that carries a "hidden meaning", as a riddle to be solved. Unlike Aarseth, who worries about the description of the text's mechanical organization as a vehicle to convey meaning, Iser (1994) understands that meaning is not something transmitted from a sender to a receiver, but "the product of an interaction between textual signs and the acts of understanding of the reader"3 (Iser, 1994: 9). For him, who proposes an explanation compatible with Dewey's theory, the literary communication processes involve a type of communication in which there is no common code a priori between interlocutors, and this code would emerge during the process: it would be up to the reader to discover the underlying code to each text.

To be more precise, we can say that, according to Iser, the text has two "codes". The "first code", consisting of the text's well established conventional structures, serves to provide the reader with directions so a "second code" can be deciphered. It is mainly from this deciphering activity that the reader derives aesthetic pleasure. Textual strategies lead the reader in this process, operating in two ways: by selection and combination.

Through the selection of one or more conventions is created a foreground/background system; the background is the system from which the norm is extracted, and the foreground, the norm extracted from its context and given additional meanings. In the literary text, the background is the repertoire, which is variable as it is not given but assumed by the text, and the foreground/background relationship in which it belongs is dialectic, because the familiar element has its meaning restructured by the unfamiliar.

Meanwhile, the combination of the elements from the repertoire highlighted by the text is what produces new meanings. Selection is an external connection; combination is internal. Although this combination is made by the author, the meaning emerges only from the synthesis made by the reader himself. Deciphering of the "second code", then, consists in the reader seeking the "coherence" in the correlation between signs, established by the author in the processes of selection and combination. To each grouping of signs produced in this process Iser gives the name of Gestalt, which he regards as the perceptual noema of the text.

The inaugural break (Dewey), or the discrepancy between the author's code and the reader's (Iser), can be interpreted, in accordance with Pareyson's views, as originated in the artist's dialogue with the matter of the work. In Small Worlds this break can be identified in the game's graphical aspects, but that does not mean that the matter of games is limited to the image. Elements such as rules and goals, as well as the fictions and narratives encoding these rules and objectives, can be counted as part of the matter of the video game. It is worth noting that these are elements with strong conventional character: game mechanics often repeat themselves, setting up game genres, and the familiarity that results from the repetition of both the mechanics and the narrative themes and fictional elements allows the player to learn how to play with ease. "Breaking" with these conventions is also breaking with the player's expectations.

In Grey's case, it can be argued that we have a puerile narrative and a twist that has been transformed into cliché by Hollywood cinema, the reveal at the end of the story

\footnotetext{
${ }^{3}$ Free translation from the authors.
} 
that the hero was just a spirit. Still, one has to realize that Grey does accomplish what Iser defines as a character of literary aesthetic experience: the deviation of an expectation of the reader, leading him to actively engage with the elements of the work to restore consistency between them. The conventions of the "platform" genre, with its mechanics and its typical themes, configure an initial horizon of expectations that leads toward a certain interpretation, but is shifted gradually by the game. The "breaks" presented challenge the first reading, leading the player to reflect on aspects of the game mechanics and narrative, seeking to regroup them in a new organization that makes sense. Thus the meaning is constructed by the player during the play, from guiding elements and strategies presented by the game.

\section{FindiNG MEANING IN THE RULES OF THE GAME}

In Small Worlds' case, it is remarkable how meaning is constructed from the internal correlation between the elements of the work, whose consistency must be synthesized by the reader, acknowledging the logic behind the selections and combinations, as pointed Iser. In the diegetic level, the player has gradual access to some information. His avatar is in a kind of space station, destroyed and deserted, and from that station it can access four other settings (Figure 2): an asteroid field, perhaps debris from the explosion of a larger body; an arctic environment with an abandoned underground military bunker, where a map can be seen with several points flashing and two remaining missile among several unoccupied silos; an empty city overtaken by water and sewage, spouting wildly through the ruins; and a cave that resembles the rotting guts of a giant dead creature, with structures that seem like bones and ribs. In each of these four scenarios the player must find an item, possibly an energy source that he can use to activate the escape pod, and finally leave the abandoned space station. From the combination of these elements, which can only be recognized in relation to a repertoire built from science fiction movies or games, the player may make a picture of Small Worlds' background narrative: maybe the hero is the last survivor of a devastating nuclear conflict, possibly triggered by the threat of a giant monster. Taken in connection with the game's mechanics of gradual exploration and its pixelated visual style, selections that refer to raster graphics and games of the first decades of the video game, we can also read the game as a tribute and a nostalgic lament for the games of that era, their fantastic themes and scenery carefully constructed pixel by pixel.

In Grey, the player is led into building the game narrative in a similar manner. The author selected, for instance, the seven objects that the player must find (balloon, clover, flower, teddy bear, necklace, bowtie and lollipop) in order to create a foreground/background connection with an established repertoire, according to which the items are perceived as signs of a relationship of love or affection between a man/boy and woman/girl. The choice for the platform game mechanics and command scheme is not casual either, but works from the assumption of a repertoire of games that refer to themes such as rescuing princesses and earning a maid's love to lead the player toward an initial reading 
that will later be reversed. By adding new elements (the transparency of the protagonist, the tombstone and the disappearance of the avatar at the end), the initial consistency is put in check and the player is forced to seek a new coherent correlation.

Another game in which this system is evident, even if resulting somewhat differently, is Scott Brodie's The Beggar. Here the player controls a rectangle with yellow eyes in a brown suit: a ragged beggar in a world populated by similar creatures in colorful costumes. It is also a two-dimensional platform game in which the player can move the avatar to the right or left using the arrow keys, can beg for coins with the down arrow, buy items with the up arrow, and pick them up them using the "Z" key. "Begging" means, in this case, emanating a yellow translucent circle that, when it touches other characters, can make them produce small yellow squares, the money of the game (Figure 7).

Over time, the protagonist starts becoming gradually clearer, until a point where a "thought bubble", in the way of the comics, shows up above him with the drawing of a fish or a piece of bread (Figure 8); this means he must collect one of the two objects (using "Z") so he can return to normal. The beggar therefore needs to feed, because as he vanishes he also becomes slower, and the game ends when he completely disappears. There are two ways to nurture the protagonist. Fish can be obtained for free with the old fisherman in the left corner of the scenery. The fisherman, however, will disappear after some time. And the bread can only be purchased in a tent, and its price increases each time it is acquired.

Besides the beggar, fisherman and baker, there are also the guards: uniformed characters that can "arrest" the beggar if he uses the yellow circle in their proximity. Arresting here is equivalent to dragging the avatar to the "police station" (a blue building with bars), and fining him by taking away part of his savings. The other characters are of three types: the ones wearing green tend to donate coins more easily, while the ones on orange and red usually react badly to requests. They emanate a red circle, and if there is a cop on the radius of the circle, he will arrest the beggar. If the player begs the same character repeatedly, it can turn from green to orange and then to red.

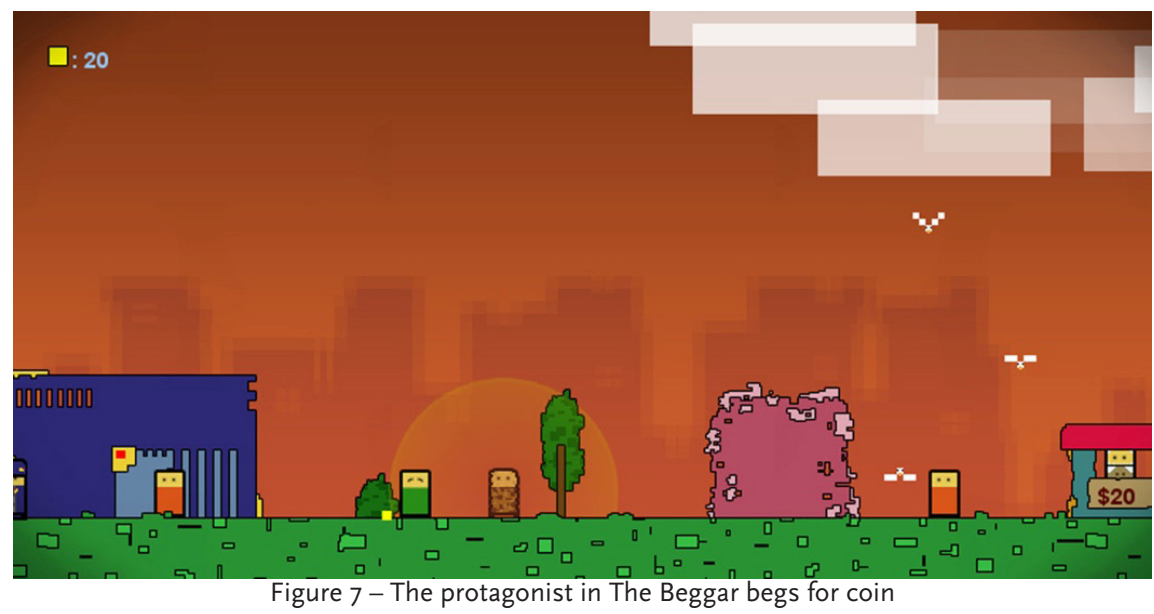




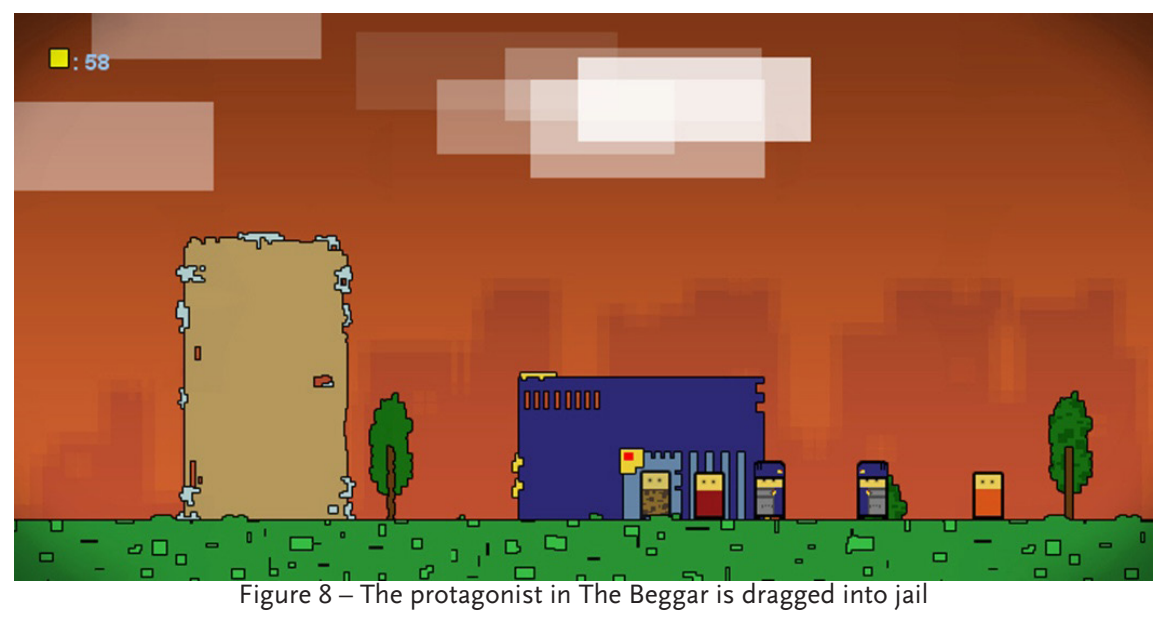

Early in the game the beggar is expelled from the right half of the scenery, which appears to be the wealthiest section of the city. He can only cross this barrier if he manages to gather two hundred pieces of coin. In this part of the scenery he can continue to beg, buy a balloon in another tent and trade it for the king's scepter at the right end of the stage; in this case, king and beggar switch roles. As king, the player can use the beg command, but with a different effect, working as an order for any policeman in reach to grab the nearest character. The "farce", however, is temporary, and soon the avatar goes back to its original appearance.

There are three possible endings: the beggar disappears, vanishing due to hunger; the beggar sits in the fisherman's boat (which can be read as a decision to leave the city, or even to become a fisherman like the old guy); and the beggar, disguised as a king, sits on the throne. We shall not focus on the possible interpretations of these endings, not only because they are open but also because they occur abruptly, without the game signaling towards them or building them as a goal. More interesting than these is the system or the logic of the fictional world that Brodie architects in the game, and the conclusions that this system allows us to reach.

The world of The Beggar is built, as in Small Worlds and Grey, from the combination of narrative elements and game aspects. One difference is that here a lot of narrative elements (themes, settings and characters) are not extracted from pre-existing narrative genres, but from today's urban social reality. The issue of begging is then presented through game mechanics such as the two-dimensional scenery, the movement scheme and the coin gathering logic typical of platform games, as well as the items purchasing system that is normally found in the RPG genre. Another difference, perhaps a more important one, is that the combination here does not result in the generation of a narrative or an interpretation of the narrative, but the creation of a stylized simulation and the correlation between that system and a real situation, which leads us to discover new things about this situation from the results that emerge from the simulation.

The Beggar's "lesson" can only be learned by playing, as the player develops more efficient strategies to achieve the objective proposed in the introduction of the game: to gather the two hundred pieces of coin needed to access the "rich" part of the town. 
After a few sessions, one can soon realize that this goal can only be achieved if the player moves quickly and manages to get the coins while evading the guards. It is enough to be arrested once or twice, and the situation deteriorates to a point where recovery becomes impossible. The money lost in "fines" cannot be regained because passersby are no longer charitable due to repeated requests; as the bread price increases just keeping the protagonist alive becomes a challenge, greatly hindering the accumulation of savings; and since most of the characters get annoyed with requests and start calling for the arrest of the beggar, he starts to lose more and more coins in fines. In the system created by Brodie, the longer the player plays, the less chance he has to accomplish the goal, and the beggar just sinks more and more inexorably into poverty.

As in the other games mentioned above, the meaning in The Beggar is produced by the player, from the conditions provided by the game, but here the meaning emerges from the dynamic system made up of the rules and objectives of the game as the interpretation of the results of a simulation - albeit a simplified and biased simulation. The player must start the game over and over several times in order to really capture the injustice of this system, which tends to aggravate the poverty of the protagonist. This, like the other games, shows that game elements - parameters, rules and possible actions can become the material of a communicative relationship akin to what was described by Pareyson, where the code is reinvented and needs deciphering in each work. Contrary to what argue some of the opponents to the recognition of the aesthetic potential of games, for whom the game aspects are obstacles to expressiveness, we have here cases where the meaning can only be deciphered from the very act of play.

\section{ASCERTAINING THE AeSTHETIC EFFECTS OF THE PLAYER'S PERFORMANCE}

Playing a digital game may seem, to the casual reader, a realization in itself of the structure of the aesthetic experience as proposed by Dewey: there is a conflict that needs to be solved by the intervention of the player; he is interested in the game elements as means for resolving this conflict; and he retrieves elements of previous experiences in order to articulate the game elements and develop strategies to remedy the breach. With the help of Pareyson and Iser, however, we can see that the conflict that Dewey refers to is not a problematic situation in the narrative, but a deviation of the code itself, and that this deviation cannot be resolved through the typical mechanical actions of most games. Instead of reflexes, aesthetic experience requires reflection; and perhaps the most valid questions regarding the possibility of the video game leading to this kind of experience is the notion that the very physical interaction typical of the medium stands in the way of this reflective activity.

From the concept of performance, however, it is possible to conceive how an action can be accompanied by reflection and have meanings or sensitive effects. According to Schechner (2006), there are three basic meanings for the word "performance". In common usage, the term refers to the success or efficiency in the execution of a task, be it professional, technical, artistic or even sexual. In the field of art it describes a specific 
artistic mode, the performance art. In a third sense, the performance can be viewed as a methodological lens that permits us to analyze events as performances. It is in this third meaning that we can apply the concept to the player's performance.

To Schechner, one of the main aspects of the concept of performance is its character as a restored behavior. Whether they are art, ritual or everyday life performances, they are always physical actions that do not occur for the first time, i. e. that are prepared or rehearsed. In Goffmann's (quoted by Schechner, 2006: 28-30) terms, they are actions that follow an established pattern he calls "routine", which can be a social convention, a theater script, or even the rules of a game. There's restored behavior even when the actor is unaware that he performs an action that is in any measure routinized, but in this performance the action gains a self-reflective character. It is because the restored behavior is marked, framed and separated that it can be articulated in a performance, calling attention to itself. Because of that Carlson (2009) defines the performance by the attitude: "we can do actions without thinking, but when we think about them, it introduces an awareness that gives them the quality of performance" 4 (Carlson, 2009: 15).

In this sense, the notion of performance can serve us here as a "methodological lens" that allows us to see certain routinized physical actions as meaning production activities in the analyzed games. In The Beggar playing becomes an experience of a reality that works by different principles than the one we live in, something that is highlighted by the difference between the system consisting of the parameters and objectives of the game and the logic of genres with which it dialogues. The game puts itself against the player in the manner how the parameters are programmed to gradually hinder the proposed objective, not in the same way that games in general operate with a gradual increase of the challenge, but in the form of an exponential curve towards the impossibility of victory. Since the system's injustice is presented in the form of a situation that can be experienced - even if it is the experience of a fictional and stylized situation - the result is not only the realization of the difficulties faced by a person who lives like that character but also the sensory experience of the situation, manifested in the frustration and despair that accompany the game sessions.

Understanding playing as a performance, of course, doesn't mean simply recognizing that it causes emotions or sensations, but also realizing that by gaining a selfreflective character - i.e., when they are accompanied by thought about the play itself - these emotions and sensations are associated with the work as its meaning, as an aesthetic effect produced by the planned structure of the experience. The aesthetic experience is not just about feeling the emotion, but also about recognizing that this emotion was communicated by the work, which was the result of the poetic operation of the artist and can be shared by others who experience the work.

According to Dewey, we react to sensory perceptions "with affective, creative and intellectual values extracted from ourselves"5 (Dewey, 2010: 242). In this sense, not only

\footnotetext{
${ }^{4}$ Free translation from the authors.

${ }^{5}$ Free translation from the authors.
} 
the construction of meaning happens through the retrieval of past experiences, but also the affective arousal. When Grey builds his narrative in the manner it does, leading the player to trigger his repertoire of experiences (not only the narrative, but also the personal ones), it creates a channel through which the twist that turns the game into a narrative about loss can find echo in the player's experience. Since the loss of a loved one is an almost universal experience, the game resonates directly with the load of experience of the players, resulting in descriptions as passionate as user Jonny_S', posted in the comments section of the page about the game:

Right away I could see that the girl was not just sitting there, but lost in thought. Since the character you play was moving around and active, I could see his world wasn't the grey one, it was hers. The first item I found was the one by the grave (the yellow flower), which told me the character I was playing was dead, thus explaining her grey world. I didn't see it as you playing a ghost, though, but rather looking at the world through her eyes. At first, all she can think about is him. Everything else is stark and immobile, but he is still so very real. Gradually, though, sparks of colour come back as she sees there is still joy. He fades out of her mind, slowly, as other things begin to come into focus. By the end, he disperses into everything around her, because even though she isn't overwhelmed with sorrow anymore, everything she sees has a hint of him in it. She could never look at a blue lake without thinking of the necklace he gave her, or see an orange sunset without thinking of the teddybear he had. This game captures amazingly, to me, the feeling of losing someone you love, moving on, and yet never having them be truly gone.

Jonny_S' reading is only possible thanks to the way the game engages elements of the repertoire in the production of meaning, with emphasis to the role of a personal repertoire in this process. The result is a direct charge of emotion that does not stem only from the sensations caused by the game mechanics or narrative, but is evoked from past experiences and adhered to these elements. Thus, emotion and meaning emerge as inseparable from each other in the effect produced by the game. Small Worlds operates under a similar logic, associating the meaning of the work to the nostalgia for the games it celebrates, by evoking the sensations produced by these old games and their pixelated graphics.

Another work that depends on the emotions and sensations caused by old games is Dys4ia, an autobiographical game created by transsexual Anna Anthropy, where she recounts her personal experience with hormone therapy. The game's title refers to gender dysphoria, another name given to the controversial notion of gender identity disorder.

Dys4ia is divided into four chapters or "levels", each consisting of a story that combines text and small games related to issues that the author discusses. Since the thread that guides it is the verbal report, the work could be classified as a case of "interactive narrative" with secondary elements typical of the Video Game. However, these elements 
play a crucial role in the production of meaning through the work, even if its definition as a game may be questioned.

In Dys4ia, the author narrates her experiences through short sentences, which are accompanied by mini-games - small interactive pieces equally short and simple, which evoke meanings and sensations from their mechanics and their dialogue with classic game mechanics. The work begins with the phrase "I feel weird about my body." At the same time, the player takes control of an angular and abstract avatar, its form refering both to the first human figures represented in pioneer arcade games and to a piece of the Tetris game (Figure 9). The background, a sort of brick wall with a space in which the part could be fitted, also evokes the mechanics of Tetris, according to which it is necessary to fit each new piece in the space without leaving gaps. In this mini-game, the player realizes what to do from the reference to the classic game, but soon sees that it is impossible to fit the piece in the given space, a result that reinforces the meaning of the original sentence. In Dys4ia, the author uses familiar elements (the gameplay mechanics) as a metaphor for the situation of the protagonist, causing the feeling evoked by the mini-games to associate to the situations described. Since the work is an experience that is unfamiliar to much of the public - it certainly isn't a recurring narrative theme, especially in games - the combination of familiar verbal report and game mechanics results in empathy, as the player identifies with the referenced games and relates the sensations evoked with the narrated experience, even if he can't directly relate with the experience.

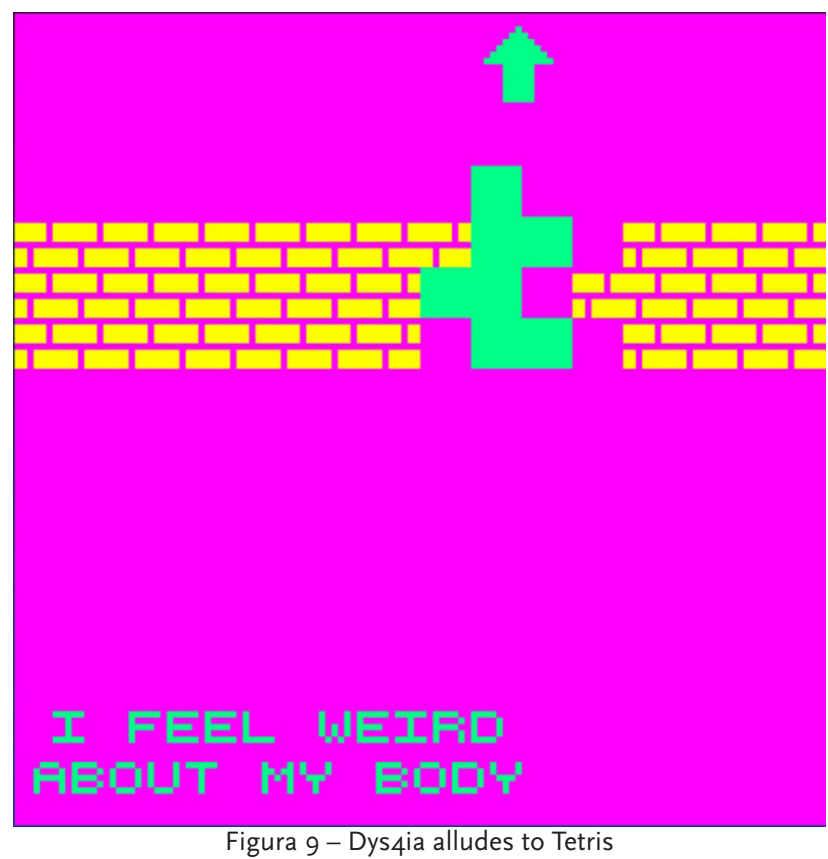

There are several different mechanics among the mini-games that make up the work. In many of them, as in the case of the block that doesn't fit, meaning is produced from the frustration of the proposed objective, as in the mini-game where the player tries to help the character to dress, while the caption explains that "girly clothes don't fit." In others, such as the one in which the player must help her find a clinic that meets her 
expectations (Figure 10), the mechanics serve to highlight the difficulties and obstacles faced by the author.

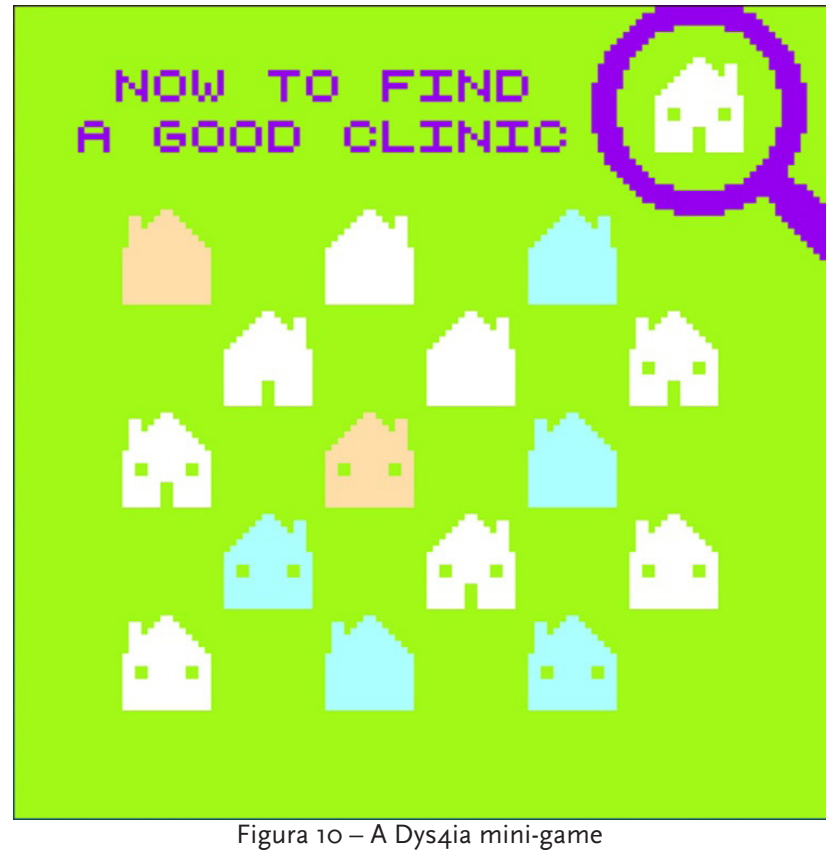

Several mini-games talk to one another, or are retrieved in different "levels" of the game with new meanings. It is the case of the one accompanied by a caption that states that "these feminists don't accept me as a woman": here the player controls an icon shield-shaped; stylized feminine "mouths" shoot speech bubbles containing the symbol for Mars, identified with the male genre, and the player must rebut these "attacks" as a racket hitting the ball, in clear reference to the Pong game. Later, the same mechanics are repeated in a scene that says: "everything my girlfriend says makes me cry." But here the speech bubble contains a question mark, and instead of reflecting the projectile back, the shield flashes when struck (a video game convention denoting damage suffered).

The initial "puzzle" is one of the recurrent scenes. At level 3, during hormone treatment, the same mechanics reappear with the caption "I feel stranger about my body than I ever did," and the block that represents the protagonist's perception of her body seems even more misshapen in respect to the area in which it should fit. At the end of the game, however, the mini-game returns, but with no caption: only the block, now switching quickly between different configurations, none of them fitting into the space. The scene serves as a nice conclusion to the story: Anna's body probably will never meet the expectations generated by the social perception of gender, but due to increased selfesteem and confidence, brought by the treatment and reported in previous scenes, she no longer feels "strange".

It is important to recognize that aesthetic experience is not about just conferring emotion to the experience of the work by evoking a repertoire, but a two-way relationship in which new experience also questions the validity of established rules and conventions. It is the mutual reorganization described by Iser, where past experiences are evoked to 
form the aesthetic experience (they give body and suggestiveness to the experience, according to Dewey), but are also changed by the relations established with the elements of the work.

By way of closing, we should point out that in the examples discussed, we tried to demonstrate an alternative analytical way to the narratologists' view, which tends to describe the video game via the metaphor of text as world. In the theoretical articulation that we presented, there is evidence of another approach - in which the notion of virtual relates to the "potential", not the "fictional" - the text as game. This metaphor is at the heart of theories such as Iser's, who conceives the text as an incomplete object that must be updated by the reader.

We must, of course, understand these expressions for what they are - metaphors — and handle them accordingly: the relationship between "text" and "game", for example, can range from literal (text is game in the mystery novels where there is a puzzle to be solved) to vague (where the concept is reduced to its essence: an activity governed by rules and exercised for pleasure). What is common to all applications of the game metaphor seems to be the notion that reading should be recognized as an interactive relationship of self-reflective character with the signs that make up the text.

Despite distancing us from the narratologists, this analytical option does not bring us close to the current traditionally opposed to this line of thought, that of ludologists because they choose only the literal application of the game concept and would refuse the application of the text metaphor. In addition, those using the text as a concept also do it in a more literal manner than we: when Aarseth claims to understand the cyberreader as someone "more integrated" in the text than the traditional reader, he in fact only admits a greater appreciation of the physical interactions possible to the first - to explore, to get lost, to discover secret paths - over the mental performance of the second, who carries out such "actions" only metaphorically. Aarseth would probably criticize our lack of formal precision and see a sort of theoretical imperialism in our adherence to theories that were not originally formulated with the differences introduced by digital media in mind, but his descriptive approach of the mechanical organization of the text, by treating it as an object rather than as an event or experience, would be seen in the light of Iser and Dewey's formulations as unable to explain how the production of meaning and aesthetic effects take place.

As the examples analyzed show us, we are dealing here with two levels of metaphors: games that are texts which are games. This means that not every game can be described as a "text that is a game", although it may be said that there is a potential affinity between games and the aesthetic experience as described by Iser and Dewey. Still, investigating the medium by the metaphor of text as game and the notion of performance allows us to overcome some of the major obstacles placed before the recognition of the video game as art by showing that, contrary to what is commonly said, being a game is no obstacle to the aesthetic experience. 


\section{REFERENCES}

Aarseth, E. (1997) Cybertext: Perspectives on Ergodic Literature, Baltimore: The John Hopkins Press.

Carlson, M. (2010) Performance: Uma Introdução Crítica, Belo Horizonte: Editora UFMG.

Castañares, W. (2007) "Cultura Visual y Crisisde laexperiência”, CIC Cuadernos de Información y Comunicación, 12 (1), available at http://revistas.ucm.es/index.php/CIYC/article/view/CIYCo707110029A, consultado em $08 / 10 / 2011$.

Dewey, J. (2010) Arte como Experiência, São Paulo: Martins Fontes.

Iser, W. (1994) The Act of Reading: A Theory of Aesthetic Response, Baltimore: The John Hopkins Press.

Murray, J. (2003) Hamlet no Holodeck: O Futuro da Narrativa no Ciberespaço, São Paulo: Itaú Cultural, Unesp.

Pareyson, L. (1997) Os Problemas da Estética, São Paulo: Martins Fontes.

Ryan, M-L. (2001) Narrative as Virtual Reality.Immersion and interactivity in Literature and Electronic Media, Baltimore: The John Hopkins University Press.

Schechner, R. (2006) "What is Performance?", in Schechner, R. (2006) Performance Studies: An Introduction, New York \& London: Routledge, pp. 28-51.

Tatarkiewicz, W. (1997) Historia de Seis Ideas. Arte, Belleza, Forma, Creatividad, Mimesis, Experiencia Estética, Madrid: Tecnos.

\section{LUDOGRAPHY}

Alexey Pajitnov (1984), Tetris $®$

Armor Games (2011), Grey

David Shute (2009), Small Worlds

Newgrounds (2012), Dys4ia

Nintendo (1981), Donkey Kong®

Scott Brodie (2009), The Beggar

\section{Bio NOTES}

Carlos Magno Camargos Mendonça - Professor at the Post Graduation Program in Comunication (PPGCOM) of the Faculdade de Filosofia e Ciências Humanas - Fafich/ UFMG (Belo Horizonte, Brasil)

E-mail: macomendonca@gmail.com

Faculdade de Filosofia e Ciências Humanas - Fafich/UFMG, Av. Antônio Carlos, 6627 - Campus Pampulha - CEP: 31270-901 - Belo Horizonte, MG -Brazil 
Filipe Alves de Freitas - Doctoral student at the Post Graduation Program in Comunication (PPGCOM) of the Faculdade de Filosofia e Ciências Humanas - Fafich/UFMG (Belo Horizonte, Brasil)

E-mail: filipealvesdefreitas@gmail.com

Faculdade de Filosofia e Ciências Humanas - Fafich/UFMG, Av. Antônio Carlos, 6627 - Campus Pampulha - CEP: 31270-901 - Belo Horizonte, MG -Brazil

* Submitted: 30-11-2014

* Accepted: 15-3-2015 